\title{
Improved Newton-Raphson digital image correlation method for full-field displacement and strain calculation
}

\author{
Corneliu Cofaru, ${ }^{1,2, *}$ Wilfried Philips, ${ }^{1}$ and Wim Van Paepegem ${ }^{2}$ \\ ${ }^{1}$ Telin-IPI-IBBT, Ghent University \\ St-Pietersnieuwstraat 41, B-9000 Ghent, Belgium \\ ${ }^{2}$ Department of Materials Science and Engineering, Ghent University \\ St-Pietersnieuwstraat 41, B-9000 Ghent, Belgium \\ ${ }^{*}$ Corresponding author: Cornel.Cofaru@telin.ugent.be
}

The 2-D inplane displacement and strain calculation problem through digital image processing methods has been studied extensively in the last three decades. Out of the various algorithms developed, the Newton-Raphson partial differential correction method is the best performing quality-wise and most widely used in practical applications despite its higher computational cost. The work presented in this paper improves the original algorithm by including adaptive spatial regularization in the minimization process used to obtain the motion data. Results indicate improvements in the strain accuracy for both small and large strains. The improvements become even more significant when employing small displacement and strain window sizes making the new method highly suitable for situations where the underlying strain data presents both slow and fast spatial variations or contains highly localized discontinuities. (c) 2010 Optical Society of America

OCIS codes: 100.2000, 100.4999, 110.4153, 110.6150

\section{Introduction}

Digital image correlation (DIC) methods gained wide acceptance in the field of experimental mechanics as a reliable tool for the full-field measurement of displacements and strains. Since their introduction [1-4] various classes of algorithms were developed, the most prominent of these involving the Newton-Raphson method of partial differential correction [5-7]. When compared to the other methods $[8,9]$ it shows higher sub-pixel accuracy and allows the reliable use of the more complex linear and quadratic local motion models at the cost of increased computational complexity and sensitivity to the interpolation method used in the minimization process. The adoption of the method has grown due to its quality advantages despite the higher computational requirements. The latter are becoming less problematic because of the continuous evolution of computing hardware performances and the fact that DIC methods are usually employed offline. The use of 
interpolants such as high order splines [10] largely diminishes the negative impact of interpolation in the final motion and strain estimates.

A fundamental limitation common to all DIC methods is the difficulty to accurately capture both high and low spatial frequency variations of the underlying displacement and strain fields. This is strictly correlated with the basic operating principle of all DIC algorithms: two images of the analyzed material specimen, taken before and after the deformation process are each divided into blocks and motion is calculated by matching the corresponding blocks from the two images. Regardless of the correlation criteria that can be used [11] in the registration process, accuracy is influenced by the size of the blocks into which the image is partitioned. If large blocks are used, slow spatial variations in the motion fields are accurately captured however, faster ones are smoothed. Smaller blocks can capture fast spatial variations as long as the assumed motion model inside the block fits locally the real displacements but the accuracy for low frequency displacement variations is negatively affected since less data is used. This paper addresses these shortcomings by extending the original Newton-Raphson method through adaptive regularization in the form of robust spatial estimators associated with each displacement component. Compared to the original method, this allows neighbouring motion information to contribute to the motion estimates in an adaptive way set by the robust estimator. As a consequence, in smooth areas of the motion field most of the neighbouring information is processed while when presented with fast variations, the algorithm selects only relevant data resulting in increased motion and strain accuracy. The theoretical aspects of the new method are presented in Section 2 with the results and conclusions in Sections 3 and 4 respectively.

\section{Newton-Raphson with robust spatial regularization}

Regularization methods have been extensively used in motion estimation problems [12-19] as a way of solving ill-posed problems by including additional spatially neighbouring data into the minimization process of a certain given energy functional. The new regularization energy functional measures the fit of a "reference" block $f(x, y)$ in the image that contains the analyzed material specimen before deformation to a "deformed" block $g\left(x^{\prime}, y^{\prime}\right)$ in the image showing the specimen after deformation. Both blocks are of size $M \times M$ pixels and $x^{\prime}=x+u(x, y), y^{\prime}=y+v(x, y)$ with:

$$
\begin{aligned}
& u(x, y)=P_{1}+P_{3}\left(x-x_{0}\right)+P_{5}\left(y-y_{0}\right) \\
& v(x, y)=P_{2}+P_{4}\left(x-x_{0}\right)+P_{6}\left(y-y_{0}\right)
\end{aligned}
$$

where $u(x, y), v(x, y)$ are the horizontal and vertical displacements of the pixel located at $(x, y)$ inside the reference block of center coordinates $\left(x_{0}, y_{0}\right)$ and $\mathbf{P}=\left(P_{i}\right)_{i=1 \ldots 6}$ is the first order displacement component vector. Using these notations, the new functional becomes:

$$
E(\mathbf{P})=E_{D}(\mathbf{P})+\lambda E_{S}(\mathbf{P})
$$


where $\lambda$ is a parameter that adjusts the strength of the regularization, $E_{D}$ is the image data fit term implemented on the sum of square differences (SSD):

$$
E_{D}(\mathbf{P})=\sum_{x=1}^{M} \sum_{y=1}^{M}(f(x, y)-g(x, y, \mathbf{P}))^{2}
$$

and $E_{S}$ is the newly added regularization constraint term:

$$
E_{S}(\mathbf{P})=\sum_{i=1}^{6} \sum_{j \in N_{i}^{8}} \rho\left(r_{i j}, \sigma_{i}\left(r_{i j}\right)\right)
$$

The regularization term from Eq. 5 is based on the Geman-McClure robust function $\rho(r, \sigma)=$ $r^{2} /\left(r^{2}+\sigma\right)$ and adaptively regulates how each displacement component $P_{i}$ is influenced by the values found in its 8-connected neighbourhood $N_{i}^{8}$ through the outlier rejection parameter $\sigma_{i}$. The six $\sigma_{i}$ parameters associated with the displacement components are calculated as a multiple of the standard deviation $\tilde{\sigma}_{i}$ of the smoothness residuals $r_{i j}=\left\{P_{i}-P_{j} \mid i=1 \ldots 6, j \in N_{i}^{8}\right\}$ and updated at each iteration in the minimization process. For each displacement component, the influence of its neighbours is proportional to the first derivative $\partial \rho(r, \sigma) / \partial r$ of the robust function seen in Fig. 1 which decreases towards zero when $\left|r_{i j}\right|>\left|\sigma_{i}\right|$. Furthermore, small $\sigma_{i}$ values, equivalent to a large degree of smoothness in the area around the displacement component currently estimated, lead to strong regularization using the closest neighbouring values. Alternatively, larger values reflect strong changes in the displacement component's neighbourhood, the influence that the neighbours exert on the component being limited and more evenly distributed. In the current implementation

$$
\sigma_{i}= \begin{cases}7 \tilde{\sigma}_{i}, & \text { if } i<3 \\ 3 \tilde{\sigma}_{i}, & \text { if } i \geq 3\end{cases}
$$

uses a higher multiplication factor of the local standard deviation for the translational components $P_{1}$ and $P_{2}$ because in most cases they represent the largest component of the displacement vector and hence differences between neighbours are larger necessitating a more relaxed or flat robust influence function.

The minimization of Eq. 3 through the Newton-Raphson iterative method has the solution at the $k$-th iteration of the form:

$$
\mathbf{P}^{(k)}-\mathbf{P}^{(k-1)}=-\frac{\nabla E\left(\mathbf{P}^{(k-1)}\right)}{\nabla \nabla E\left(\mathbf{P}^{(k-1)}\right)}
$$

where

$$
\nabla E(\mathbf{P})=\left[\left(\frac{\partial E_{D}}{\partial P_{1}}+\lambda \frac{\partial E_{S}}{\partial P_{1}}\right) \cdots\left(\frac{\partial E_{D}}{\partial P_{6}}+\lambda \frac{\partial E_{S}}{\partial P_{6}}\right)\right]
$$


and

$$
\nabla \nabla E(\mathbf{P})=\left[\begin{array}{ccc}
\left(\frac{\partial^{2} E_{D}}{\partial P_{1}^{2}}+\lambda \frac{\partial^{2} E_{S}}{\partial P_{1}^{2}}\right) & \cdots & \left(\frac{\partial^{2} E_{D}}{\partial P_{1} \partial P_{6}}\right) \\
\vdots & \ddots & \vdots \\
\left(\frac{\partial^{2} E_{D}}{\partial P_{6} \partial P_{1}}\right) & \cdots & \left(\frac{\partial^{2} E_{D}}{\partial P_{6}^{2}}+\lambda \frac{\partial^{2} E_{S}}{\partial P_{6}^{2}}\right)
\end{array}\right]
$$

are the Jacobian and Hessian matrices of the energy functional with the regularization term partial derivatives:

$$
\frac{\partial E_{S}}{\partial P_{i}}=\sum_{j \in N_{i}^{8}} \frac{2 \sigma_{i}\left(P_{i}-P_{j}\right)}{\left(\sigma_{i}+\left(P_{i}-P_{j}\right)^{2}\right)^{2}}
$$

and

$$
\frac{\partial^{2} E_{S}}{\partial P_{i}^{2}}=\sum_{j \in N_{i}^{8}} \frac{2 \sigma_{i}^{2}-6 \sigma_{i}\left(P_{i}-P_{j}\right)^{2}}{\left(\sigma_{i}+\left(P_{i}-P_{j}\right)^{2}\right)^{3}}
$$

respectively. Note that $\partial^{2} E_{S} / \partial P_{i}^{2}$ is only present on the first diagonal of the Hessian since the second partial derivatives of $E_{S}$ with respect to two different displacement components are zero.

The method starts by dividing the reference image into blocks and finding the best match for each block in the deformed image through cross-correlation coefficient minimization. The resulting integer pixel displacements will represent the initial solution for the Newton-Raphson minimization step of the method. The latter updates at each iteration the entire motion vector field excluding the locations where convergence has already been reached. As convergence criterion, a difference smaller than $10^{-5}$ between consecutive iterations for all displacement components has been chosen. Once a motion vector reached convergence its six components will stop being updated however they will be used in subsequent iterations in calculating the regularization terms of their neighbours. If in three successive iterations the total number of motion vectors that reached convergence does not change, the algorithm stops. To increase the stability of the method, the first three iterations are executed without taking into consideration the regularization terms.

\section{Tests and results}

The evaluation of the new method consists of calculating the displacements and strains in four experimental scenarios: two numerical simulations and two real mechanical experiments. Each scenario contains an image pair composed of a "reference" and a "deformed" image. The first two scenarios correspond to a "plate with hole under biaxial stress" model [20] with their deformed images numerically obtained by warping a common reference speckle image using known displacements and radial basis function image interpolation [9]. In third and fourth experimental scenarios which represent real experiments, a plastic film specimen $25.3 \mathrm{~mm}$ in width, $250 \mathrm{~mm}$ in length and $0.1 \mathrm{~mm}$ in thickness with a lateral slit into its right edge and an aluminum specimen $25.25 \mathrm{~mm}$ in width, $280 \mathrm{~mm}$ in length and $2.95 \mathrm{~mm}$ in thickness with two lateral slits on both edges undergo uniaxial load in a vertical upward direction. All speckle images were captured using a Pixelink PL-A782 camera at the maximum resolution of $2208 \times 3000$ pixels out of which regions $1024 \times 1024$ 
pixels in size were actively used in the tests. Through the experimental setup one pixel corresponds to $8.33 \mu \mathrm{m}$ in the object plane for the first two experiments, $11.92 \mu \mathrm{m}$ in the third experiment and $17 \mu \mathrm{m}$ in the fourth experiment. The camera was aligned perpendicular to the specimen surface with a laser to eliminate out of plane displacement effects and calibrated through the proprietary software to compensate for fixed-pattern noise, photo response non-uniformities and lighting variations across the specimen's surface. The machine used to deform the specimens in the last two experiments was a Instron 8801 servo-hydraulic system. In Fig. 2 the two reference images used in the first three scenarios are shown. The full camera frame as well as the reference and deformed images for the fourth scenario are shown in Fig. 12.

The deformed image in the first experimental scenario presents only subpixel underlying displacements as can be observed in Fig. 3 with small (Cauchy) strains between $1.2 \times 10^{-3}$ and $-6.86 \times 10^{-4}$ for $\varepsilon_{x x}, 2.8 \times 10^{-3}$ and $-2.8 \times 10^{-3}$ for $\varepsilon_{x y}$ and $4.7 \times 10^{-3}$ and $1.73 \times 10^{-5}$ for $\varepsilon_{y y}$. This simulates a steel plate with a horizontally applied load of $50 \mathrm{MPa}$ and a vertically applied load of $350 \mathrm{MPa}$. The set of images associated with the second scenario simulates a rubber plate under $2 \mathrm{MPa}$ horizontally and $4 \mathrm{MPa}$ vertically applied loads. Displacement and strain spatial variations are similar to the ones present in the first pair of images with amplitudes varying between 4 and -4 pixels for the horizontal displacements and 21.8 and -21.8 pixels for the vertical displacements. The associated large (Green-Lagrange) strain values vary between $4.47 \times 10^{-2}$ and $-2 \times 10^{-2}$ for $\varepsilon_{x x}, 3.84 \times 10^{-2}$ to $-3.84 \times 10^{-2}$ for $\varepsilon_{x y}$ and $9.07 \times 10^{-2}$ to $-1.02 \times 10^{-2}$ for $\varepsilon_{y y}$. For the last two experiments the exact strain values are unknown since they relate to real experimental data and do not represent numerical simulations. Several observations can be however made: in the third experiment, rigid body translation in excess of 50 pixels for the vertical displacement component and 8 pixels for the horizontal component are present due to small slips of the plastic film in the grips of the servo-hydraulic machine. The strain concentrations in this case are assumed to be present at the tip of the lateral slit and to decrease rapidly with the distance from it. Due to the calibration of the camera the random noise levels across the image are considered to be very low. In the last experiment rigid body translations due to slipping in the mechanical grips are virtually eliminated through better gripping of the aluminum specimen in the machine. Noise levels are further reduced respect to the third experiment by automatic averaging of three camera frames before and after deformation to obtain the reference and deformed images respectively.

\section{A. Quality analysis}

In the evaluation process of the first two - numerical - experiments, blocks $15 \times 15,21 \times 21$ and $27 \times 27$ pixels with a 7 pixel step size have been employed. For each block size, four values of the parameter $\lambda$ were examined: 0, corresponding to the regular Newton-Raphson approach without regularization, 50, 100 and 200. Strain calculations were done according to [21] using strain windows

$3 \times 3,5 \times 5,7 \times 7,9 \times 9$ and $11 \times 11$ motion vectors in size. This has the double purpose of assessing the combined impact of the image block size and strain window size in the overall strain accuracy and to evaluate how the new method performs under these variations compared to the classical method. 
The mean of the absolute horizontal, shear and vertical strain errors for the first experimental scenario using blocks $15 \times 15,21 \times 21$ and $27 \times 27$ pixels in size are presented in Fig. 4, Fig. 5 and Fig. 6 respectively. The results indicate large improvements in the average strain error ranging from approximately $25 \%$ to more than $50 \%$ for the smaller strain window sizes. This is to be expected as the estimates obtained using regularization include neighbouring spatial information and thus are more reliable than the non-regularized estimates. The proposed method produces the minimum errors regardless of strain windows and block sizes for the horizontal and vertical strains. In the case of the shear strains, it performs better when using strain windows smaller than $11 \times 11$. It is clearly noticeable that increases of the strain window size, image block size or both have a lesser impact in the accuracy of the method when using regularization. This suggests increased practical applicability of the proposed method in situations where these smaller sizes are required like low resolution images or localized stress concentrations. The lower limit of the mean errors remains approximately the same for all block sizes in the range of 150 to $200 \mu \varepsilon$.

The results from the second experiment synthesized in Fig. 7, Fig. 8 and Fig. 9 indicate that also for larger strains the introduction of spatial regularization improves the accuracy of the NewtonRaphson DIC method. The proposed method outperforms the classical one at all strain window and image block sizes providing also the absolute minimum errors. It is however interesting to notice that the minimum errors for all three strains were obtained using the smallest image block size of $15 \times 15$ pixels and strain windows sizes either $7 \times 7$ or $9 \times 9$ motion vectors. The negative effect of larger strain windows is becoming more evident as the image block size increases: for block sizes of $21 \times 21$ pixels and $27 \times 27$ pixels using a strain window $7 \times 7$ motion vectors produces consistently better results than a $11 \times 11$ strain window. In the case of the vertical strains in Fig. 9, strain windows sized $5 \times 5$ produce smaller errors than the ones sized $9 \times 9$ and $11 \times 11$. Besides the improvements brought by the proposed method, the results from the second experiment indicate that unless the underlying strains that are to be calculated present smooth variations which in turn require solid prior knowledge about the experimental behavior of the analyzed mechanical specimen, increasing the strain window sizes and image block sizes can lead to deterioration of the measured strain accuracy. It is thus preferable to use smaller block sizes and strain window sizes to avoid over-smoothing the strain estimates with any additional neighbouring information pooled in an adaptive manner.

The last two practical experiments are meant to be a only visual evaluation of the observations made for first two experimental scenarios since no numerical verification is possible. In the third experiment two tests were done where the regularization strength parameter $\lambda$ had the values of 0 and 1000. The value of $\lambda$ is larger than in the previous tests so that the stronger adaptive smoothing would compensate for any possible effects of camera noise present in the images on the motion estimates. The block size was fixed at $27 \times 27$ pixels with a 7 pixel step. Large strains are expected to be present at the tip of the crack and its immediate vicinity with smaller and smoothly varying strains in the rest of the material's surface. In Fig. 10 the vertical strains $\varepsilon_{y y}$ calculated with a strain window $5 \times 5$ motion vectors for the two tests are shown. The benefits of adaptive regularization are evident: the high strain concentration values located at the tip of the slit are 
maintained similar to those obtained without regularization while in the rest of the specimen, the smaller peaks which are erroneous are significantly reduced. Using larger strain windows reduces further the erroneous discontinuities across the strain fields at the cost of also lowering or smoothing the slit tip strain values: using a $9 \times 9$ strain window the amplitudes are reduced by $35 \%$ to $50 \%$. Comparing the shear strains from Fig. 11 leads to similar conclusions: the large strains around the crack tip do not undergo significant modification with respect to the non-regularized algorithm while the rest of the strain field presents smoother variations.

For the fourth and last experimental scenario, the parameter $\lambda$ took the values of 0 and 200 . The strains were calculated using image block sizes of $27 \times 27$ pixels and strain windows of $5 \times 5$ and $9 \times 9$ motion vectors. The vertical strains obtained using the two strain windows sizes can be seen in Fig. 13 and Fig. 14 with the shear strains in Fig. 15 and Fig. 16 respectively. It can be observed that the influence of the adaptive regularization term is stronger in the areas where the strain field values present spatial similarity producing smoother variations while preserving larger strain gradient areas. This can be interpreted as an elimination of the calculus noise in the areas where there is little variational uncertainty regarding the data. Using a larger strain window has a smoothing effect of the entire strain field, effect most noticeable at the peak strain values. Although it is hard to determine which strain window produces a higher overall accuracy it is easily foreseeable that increasing the strain window over a certain limit can have detrimental effects on the accuracy as seen in the results from the second experiment. Through the increases in accuracy brought, the proposed extension not only improves results in common usage scenarios but also extends the practical applicability of the method for small image resolutions and highly localized strains.

\section{B. Computational performance analysis}

The methods presented in this paper have been implemented in Matlab and executed on a Intel Core 2 Duo $2 \mathrm{GHz}$ processor with $4 \mathrm{~GB}$ of Ram running the Linux operating system. Rather than concentrate on the actual execution times which can vary depending on the machine used, the performance analysis takes into consideration the total number of iterations performed and the rate with which the number of motion vectors that did not converge yet decreases at each iteration. The performance graphs related to the first image set are shown in Fig. 17. Increasing values of the regularization strength parameter $\lambda$ have a negative impact on the total number of iterations needed to reach convergence. The influence is more evident for $15 \times 15$ pixel blocks: the method without regularization requires 25 iterations to converge while for $\lambda=50$ the number of needed iterations is 64. Using larger block sizes leads to a decrease in the number of iterations needed for convergence with 18 iterations needed to converge when regularization is not used, 34 iterations for $\lambda=50$ and 45 iterations for $\lambda=200$. The right graph in the figure shows the number of motion vectors that did not yet reach convergence given a certain iteration using a fixed block size of $27 \times 27$ pixels. The graphs start from a common point which represents the end of the third iteration (when the regularization process starts) and subsequently diverge indicating different convergence speeds. The relationship between the number of non-converging motion vectors and the number of 
iterations effectuated seems to be linear regardless of the regularization with a stronger decline in the number of converging blocks / iteration once the number of blocks remaining approaches 2000 or approximately $10 \%$ of the total number of 20449 blocks. A possible explanation is that the image information for those blocks is not entirely adequate or the displacement components are large, the method necessitating more iterations to converge. Another way of assessing the performance impact of regularization is to consider the processing that needs to be done for one block during one iteration as an elementary operation. In this way, considering again the block size fixed at $27 \times 27$ pixels, the total number of operations needed to reach convergence for $\lambda=0$ is 90092 , for $\lambda=50$ is 193141, for $\lambda=100$ is 234378 and for $\lambda=200$ is 281746 . A clear conclusion is that the speed does not decrease linearly with $\lambda$ but rather tends to saturate. The performance observations remain overall unchanged for the second set of images where larger strains are present, the only difference being an increased overhead needed to calculate the larger integer displacements.

\section{Conclusion}

In this paper an extension of the Newton-Raphson partial differential correction DIC method through the addition of adaptive spatial regularization has been presented and its accuracy and performance impact investigated. The regularization terms based on the Geman-McClure robust function adaptively integrate neighbouring information motion into the motion estimates and are recalculated at each iteration to adjust the neighbour influence according to the local spatial smoothness in each displacement component field. Results indicate accuracy improvements consisting in mean errors up to $50 \%$ smaller compared to the non-regularized method for both small and large strains. The quality advantages of the regularized method for smaller image blocks and strain windows are strongly desired since their smaller sizes increase the locality of the available deformation information. The inclusion of the regularization term increases computational cost of method which in turn is influenced by the strength of the regularization and sizes of the image blocks used in the motion estimation process. Despite the computational costs, the quality advantages brought by the proposed method make it a viable alternative to existing DIC methods.

\section{Acknowledgments}

The authors would like to acknowledge the Interuniversity Attraction Poles Program (IUAP-VI) of the Belgian Federal Science Policy.

\section{References}

1. W. H. Peters and W. F. Ranson, "Digital imaging techniques in experimental stress-analysis," Opt. Eng. 21, 427-431 (1981).

2. M. A. Sutton, W. J. Wolters, W. H. Peters, W. F. Ranson and S. R. McNeill, "Determination of displacements using an improved digital correlation method," Image Vision Comput. 1, 133-139 (1983). 
3. W. H. Peters, W. F. Ranson, M. A. Sutton, T.C. Chu and J. Anderson, "Application of digital correlation methods to rigid body mechanics," Opt. Eng. 22, 738-742 (1983).

4. T.C. Chu, W. F. Ranson, M. A. Sutton and W. H. Peters, "Applications of digital-imagecorrelation techniques to experimental mechanics," Exp. Mech. 25, 232-244 (1985).

5. H. A. Bruck, S. R. McNeill, M. A. Sutton and W. H. Peters, "Digital image correlation using Newton-Raphson method of partial differential correction," Exp. Mech. 29, 261-267 (1989).

6. G. Vendroux and W. G. Knauss, "Submicron deformation field measurements: Part 2. Improved digital image correlation," Exp. Mech. 38, 86-92 (1998).

7. H. Lu and P. D. Cary, "Deformation measurements by digital image correlation: Implementation of a second-order displacement gradient," Exp. Mech. 40, 393-400 (2000).

8. B. Pan, H-M. Xie, B-Q. Xu and F-L. Dai, "Performance of sub-pixel registration algorithms in digital image correlation," Meas. Sci. Technol. 17, 1615-1621 (2006).

9. C. Cofaru, W. Philips and W. Van Paepegem, "Evaluation of digital image correlation techniques using realistic ground truth speckle images," Meas. Sci. Technol. 21, 055102 (17 pp.) (2010).

10. M. A. Sutton, J-J. Orteu and H. W. Schreier, Image correlation for shape, motion and deformation measurements (Springer, 2009).

11. B. Pan, K-M. Qian, H-M. Xie and A. Asundi, "Two-dimensional digital image correlation for in-plane displacement and strain measurement: a review," Meas. Sci. Technol. 20, 062001 (17 pp.) (2009).

12. C-H. Teng, S-H. Lai, Y-S. Chen and W-H. Hsu, "Accurate optical flow computation under non-uniform brightness variations," Comput. Vis. Image Underst. 97, 315-346 (2005).

13. M. Ye, R. M. Haralick and L. G. Shapiro, "Estimating piecewise-smooth optical flow with global matching and graduated optimization," IEEE Trans. Pattern Anal. Mach. Intell. 25, 1625-1630 (2003).

14. G. Le Besnerais and F. Champagnat, "B-spline image model for energy minimization-based optical flow estimation," IEEE Trans. Image Process. 15, 3201-3206 (2006).

15. J-D. Kim and J. Kim, "Effective nonlinear approach for optical flow estimation," Signal Process. 81, 2249-2252 (2001).

16. M. J. Black and P. Anandan, "The robust estimation of multiple motions: Parametric and piecewise-smooth flow fields," Comput. Vis. Image Underst. 63, 75-104 (1996).

17. Y-H. Kim, A. M. Martìnez, A. C. Kak, "Robust motion estimation under varying illumination," Image Vis. Comput. 23, 365-375 (2005).

18. J. Weickert and C. Schnorr, "Variational optic flow computation with a spatio-temporal smoothness constraint," J. Math. Imag. Vis. 14, 245-255 (2001).

19. A. Bruhn, J Weickert, C. Schnorr, "Lucas/Kanade meets Horn/Schunck: Combining local and global optic flow methods," Int. J. Comput. Vis. 61, 211-231 (2005).

20. J. F. Cárdenas-García and J. J. E. Verhaegh, "Catalogue of Moiré fringes for a bi-axially-loaded infinite plate with a hole," Mech. Research Commun. 26, 641-648 (1999). 
21. B. Pan, A. Asundi, H-M. Xie and J. X. Gao, "Digital image correlation using iterative least squares and pointwise least squares for displacement field and strain field measurements," Opt. Lasers Eng. 47, 865-874 (2009) 


\section{List of Figure Captions}

Fig. 1 The Geman-McClure robust function (left) and its first derivative (right) for the shape parameter values $\sigma=0.01,0.1,1$.

Fig. 2 The reference images used the first two (left) and third (right) experimental scenarios.

Fig. 3 Contours of the horizontal (left) and vertical (right) displacement components for the first experimental scenario.

Fig. 4 Mean absolute strain errors for the horizontal (left), shear (center) and vertical (right) small (Cauchy) strains as functions of the strength regularization parameter $\lambda$ and strain window size $W$ using block sizes of $15 \times 15$ pixels.

Fig. 5 Mean absolute strain errors for the horizontal (left), shear (center) and vertical (right) small (Cauchy) strains as functions of the strength regularization parameter $\lambda$ and strain window size $W$ using block sizes of $21 \times 21$ pixels.

Fig. 6 Mean absolute strain errors for the horizontal (left), shear (center) and vertical (right) small (Cauchy) strains as functions of the strength regularization parameter $\lambda$ and strain window size $W$ using block sizes of $27 \times 27$ pixels.

Fig. 7 Mean absolute strain errors for the horizontal (left), shear (center) and vertical (right) large (Green-Lagrange) strains as functions of the strength regularization parameter $\lambda$ and strain window size $W$ using block sizes of $15 \times 15$ pixels.

Fig. 8 Mean absolute strain errors for the horizontal (left), shear (center) and vertical (right) large (Green-Lagrange) strains as functions of the strength regularization parameter $\lambda$ and strain window size $W$ using block sizes of $21 \times 21$ pixels.

Fig. 9 Mean absolute strain errors for the horizontal (left), shear (center) and vertical (right) large (Green-Lagrange) strains as functions of the strength regularization parameter $\lambda$ and strain window size $W$ using block sizes of $27 \times 27$ pixels.

Fig. 10 Vertical strains corresponding to the third experiment. The results were obtained using $\lambda=0$ (left) and $\lambda=1000$ (right), block sizes of $27 \times 27$ pixels and a strain window of $5 \times 5$ motion vectors.

Fig. 11 Shear strains corresponding to the third experiment. The results were obtained using $\lambda=0$ (left) and $\lambda=1000$ (right), block sizes of $27 \times 27$ pixels and a strain window of $5 \times 5$ motion vectors.

Fig. 12 The full frame captured by the camera (left), reference (center) and deformed (right) images used in the last experiment.

Fig. 13 Vertical strains corresponding to the fourth experiment. The results were obtained using $\lambda=0$ (left) and $\lambda=200$ (right), block sizes of $27 \times 27$ pixels and a strain window of $5 \times 5$ motion vectors.

Fig. 14 Vertical strains corresponding to the fourth experiment. The results were obtained using $\lambda=0$ (left) and $\lambda=200$ (right), block sizes of $27 \times 27$ pixels and a strain window of $9 \times 9$ motion vectors.

Fig. 15 Shear strains corresponding to the fourth experiment. The results were obtained using 
$\lambda=0$ (left) and $\lambda=200$ (right), block sizes of $27 \times 27$ pixels and a strain window of $5 \times 5$ motion vectors.

Fig. 16 Shear strains corresponding to the fourth experiment. The results were obtained using $\lambda=0$ (left) and $\lambda=200$ (right), block sizes of $27 \times 27$ pixels and a strain window of $9 \times 9$ motion vectors.

Fig. 17 Number of iterations as a function of the strength regularization parameter $\lambda$ for different block sizes (left) and the number of motion vectors that did not yet converge for a fixed block size of $27 \times 27$ pixels for different $\lambda$ values (right). 

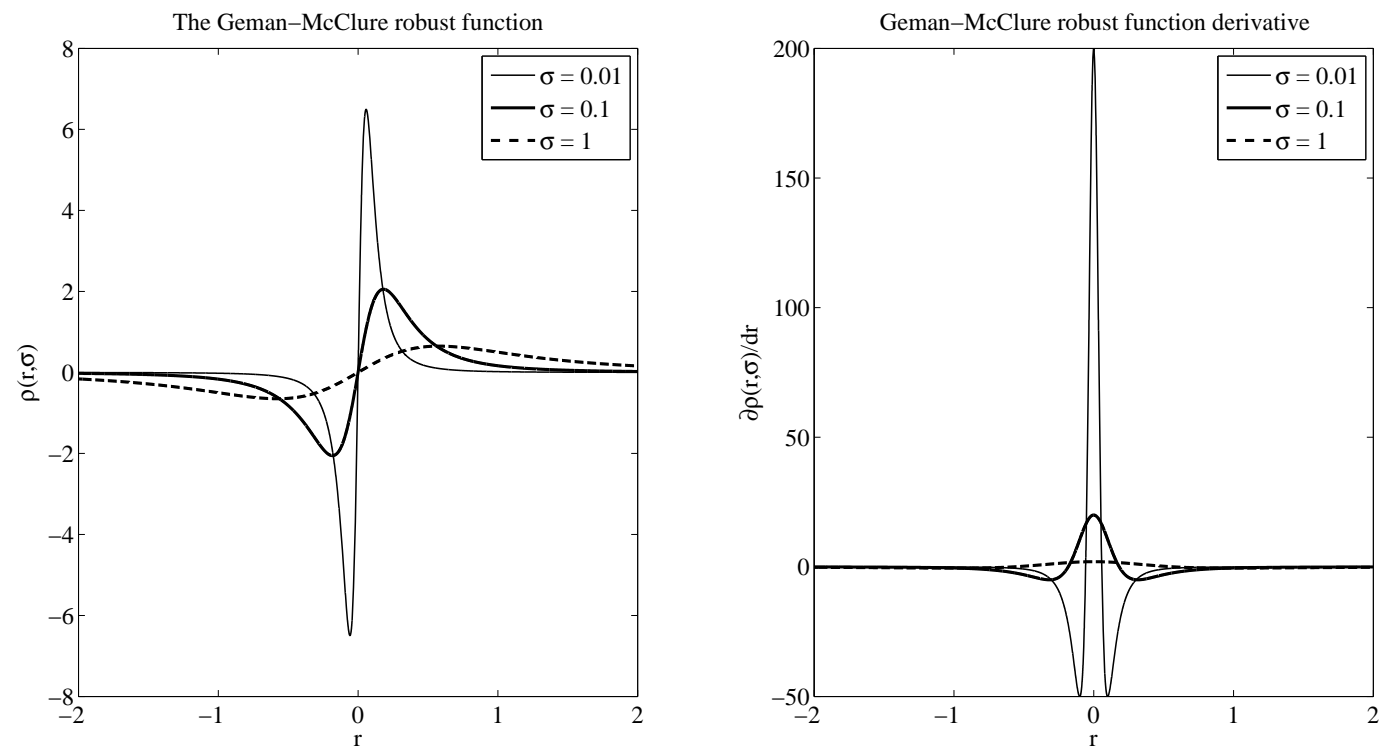

Fig. 1. The Geman-McClure robust function (left) and its first derivative (right) for the shape parameter values $\sigma=0.01,0.1,1$. F1.EPS. 
Reference image of the first two image pairs

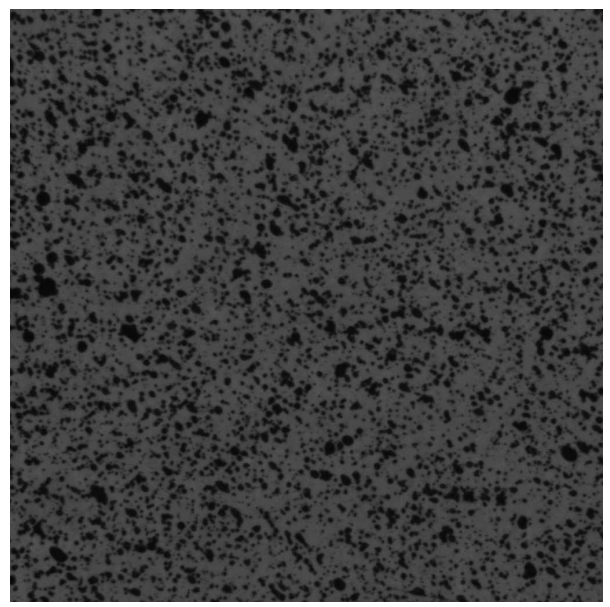

Reference image of the third image pair

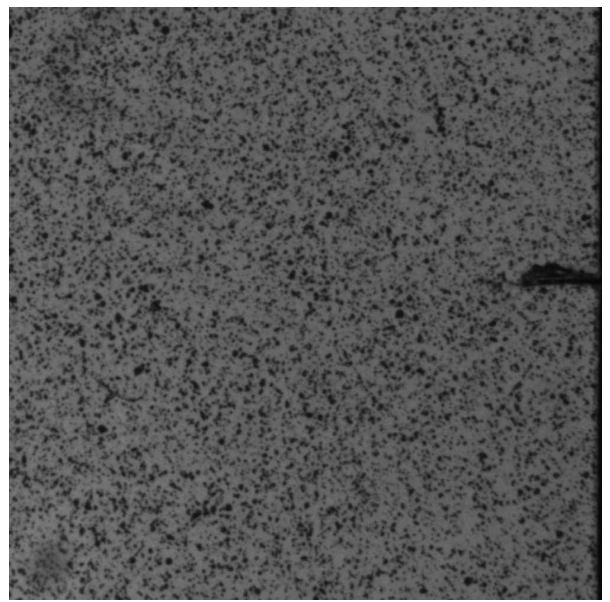

Fig. 2. The reference images used the first two (left) and third (right) experimental scenarios. F2.EPS
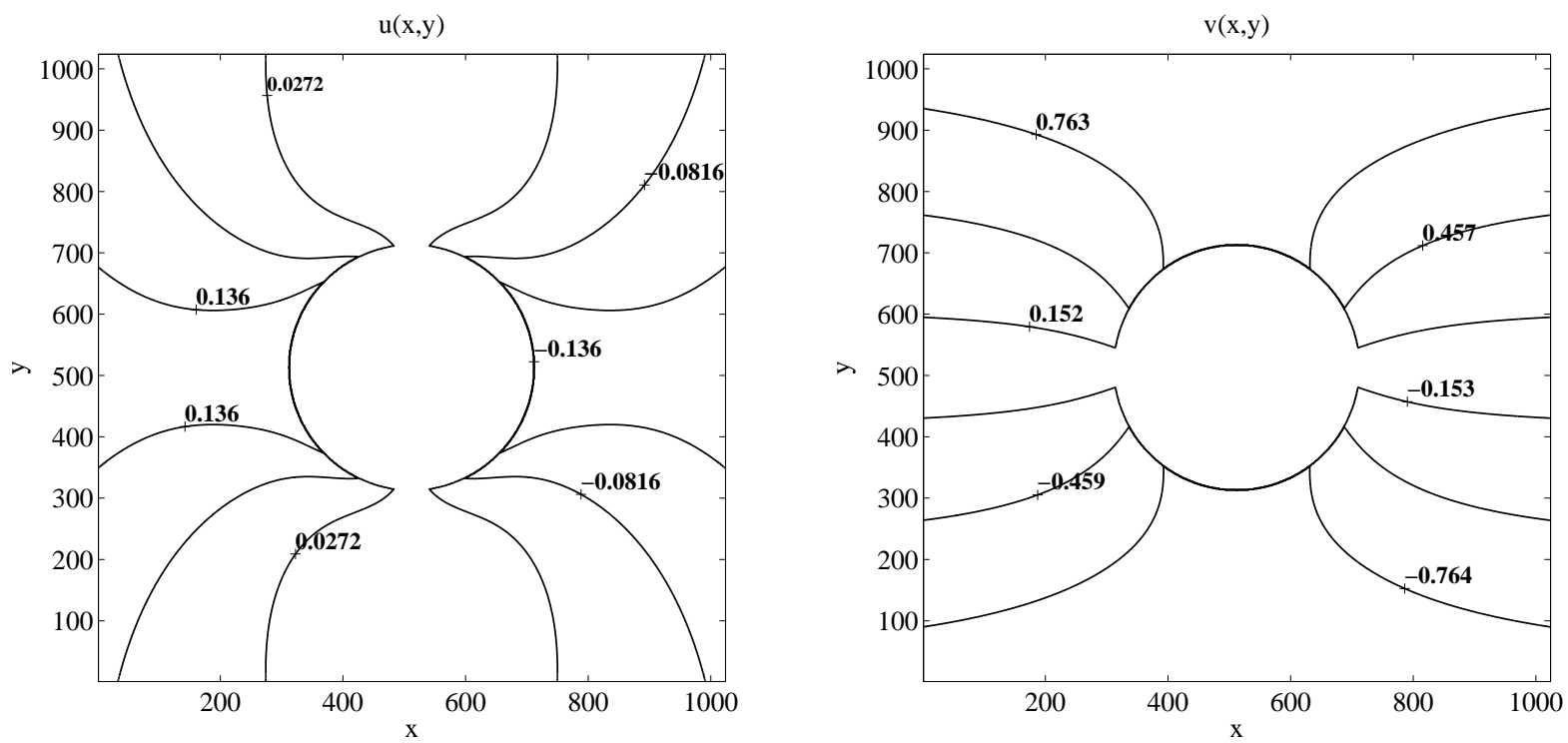

Fig. 3. Contours of the horizontal (left) and vertical (right) displacement components for the first experimental scenario. F3.EPS 


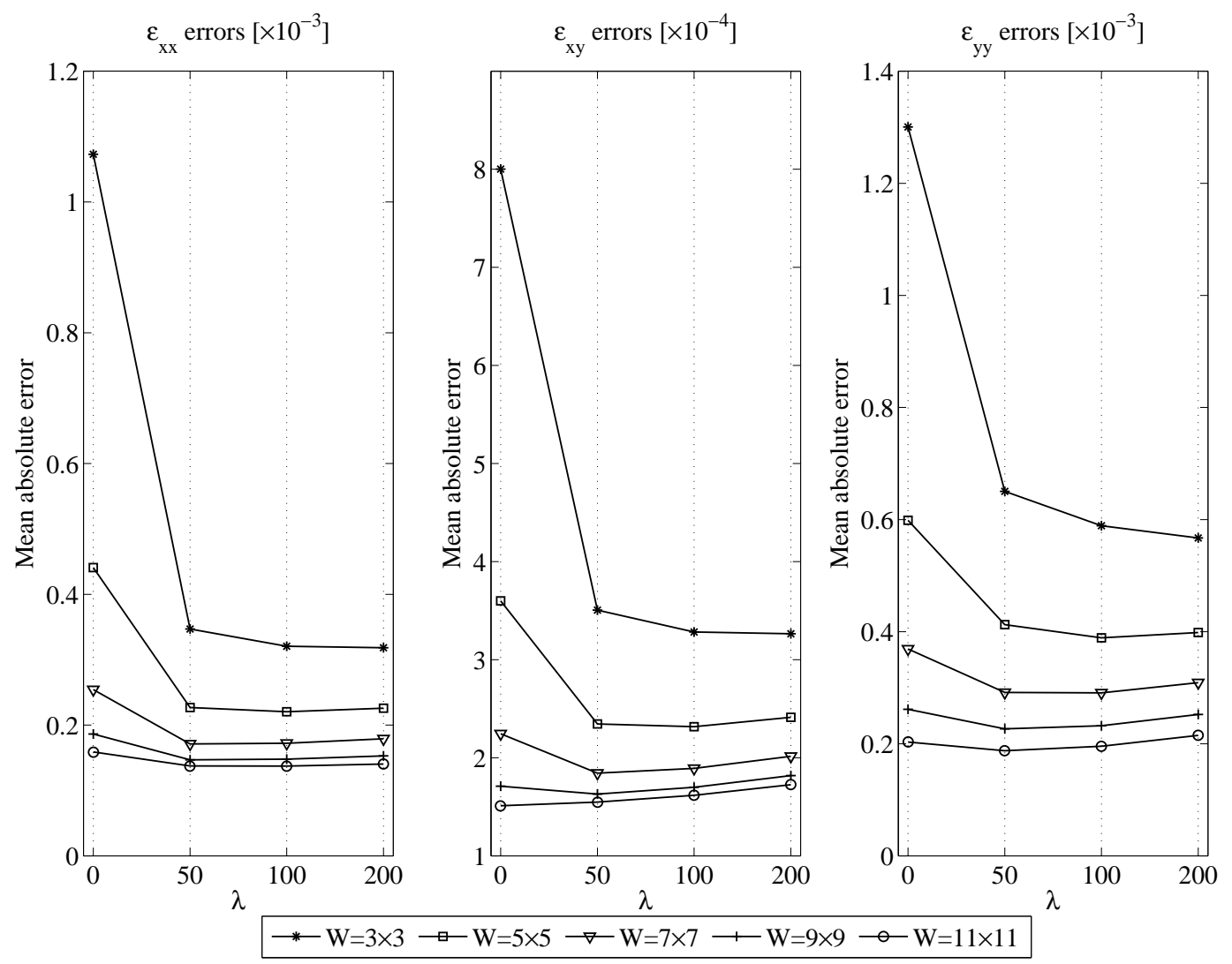

Fig. 4. Mean absolute strain errors for the horizontal (left), shear (center) and vertical (right) small (Cauchy) strains as functions of the strength regularization parameter $\lambda$ and strain window size $W$ using block sizes of $15 \times 15$ pixels. F4.EPS 


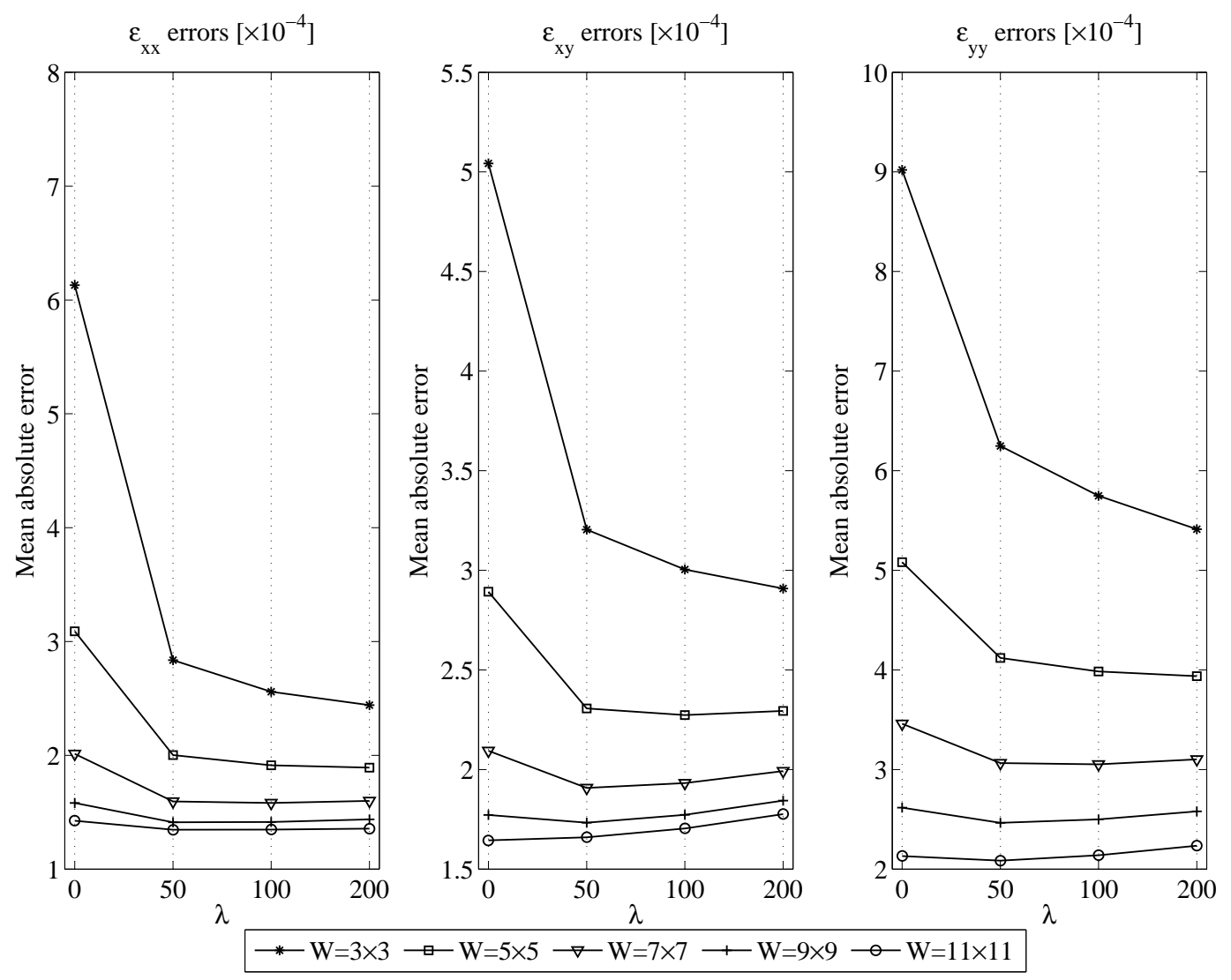

Fig. 5. Mean absolute strain errors for the horizontal (left), shear (center) and vertical (right) small (Cauchy) strains as functions of the strength regularization parameter $\lambda$ and strain window size $W$ using block sizes of $21 \times 21$ pixels. F5.EPS 


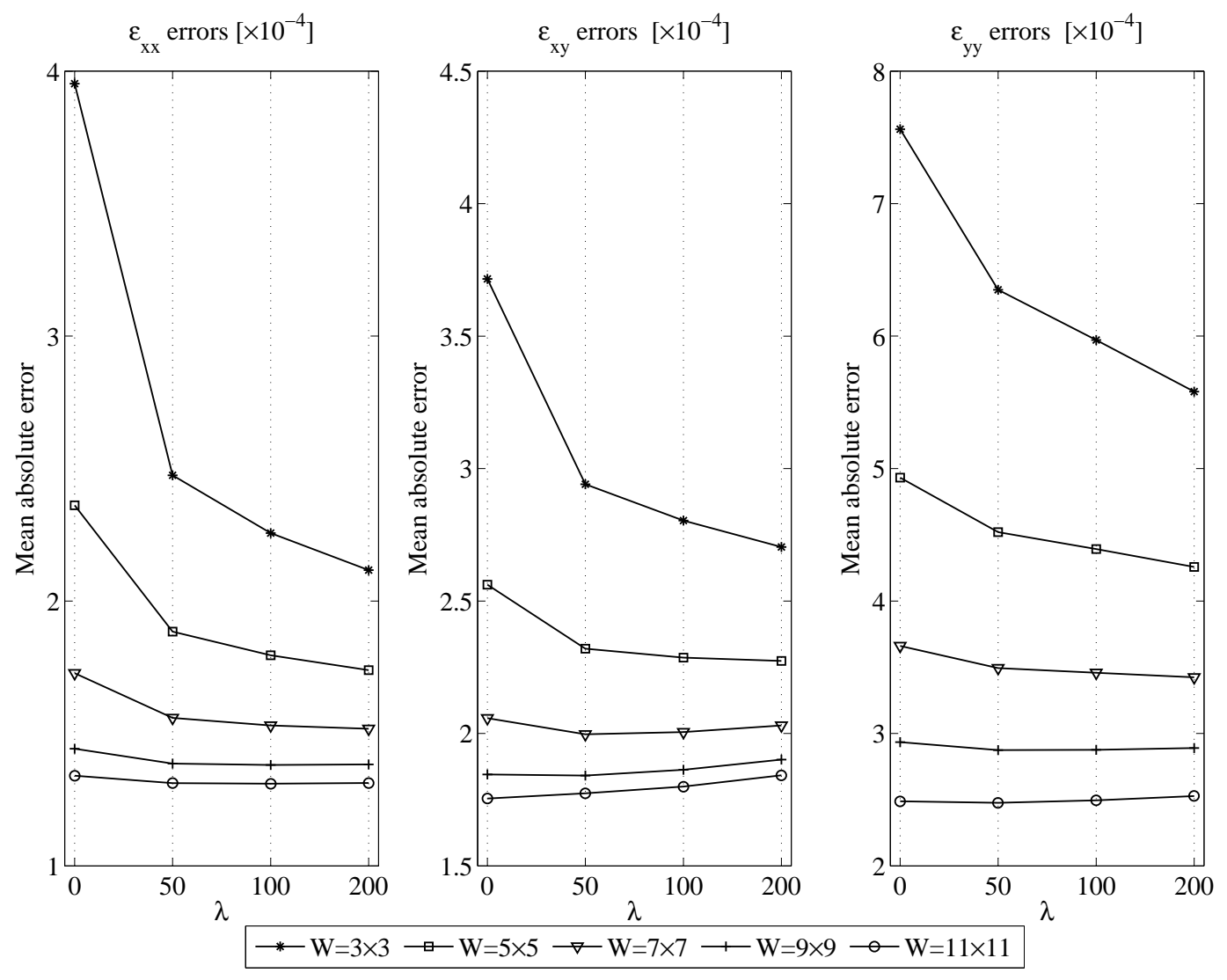

Fig. 6. Mean absolute strain errors for the horizontal (left), shear (center) and vertical (right) small (Cauchy) strains as functions of the strength regularization parameter $\lambda$ and strain window size $W$ using block sizes of $27 \times 27$ pixels. F6.EPS 


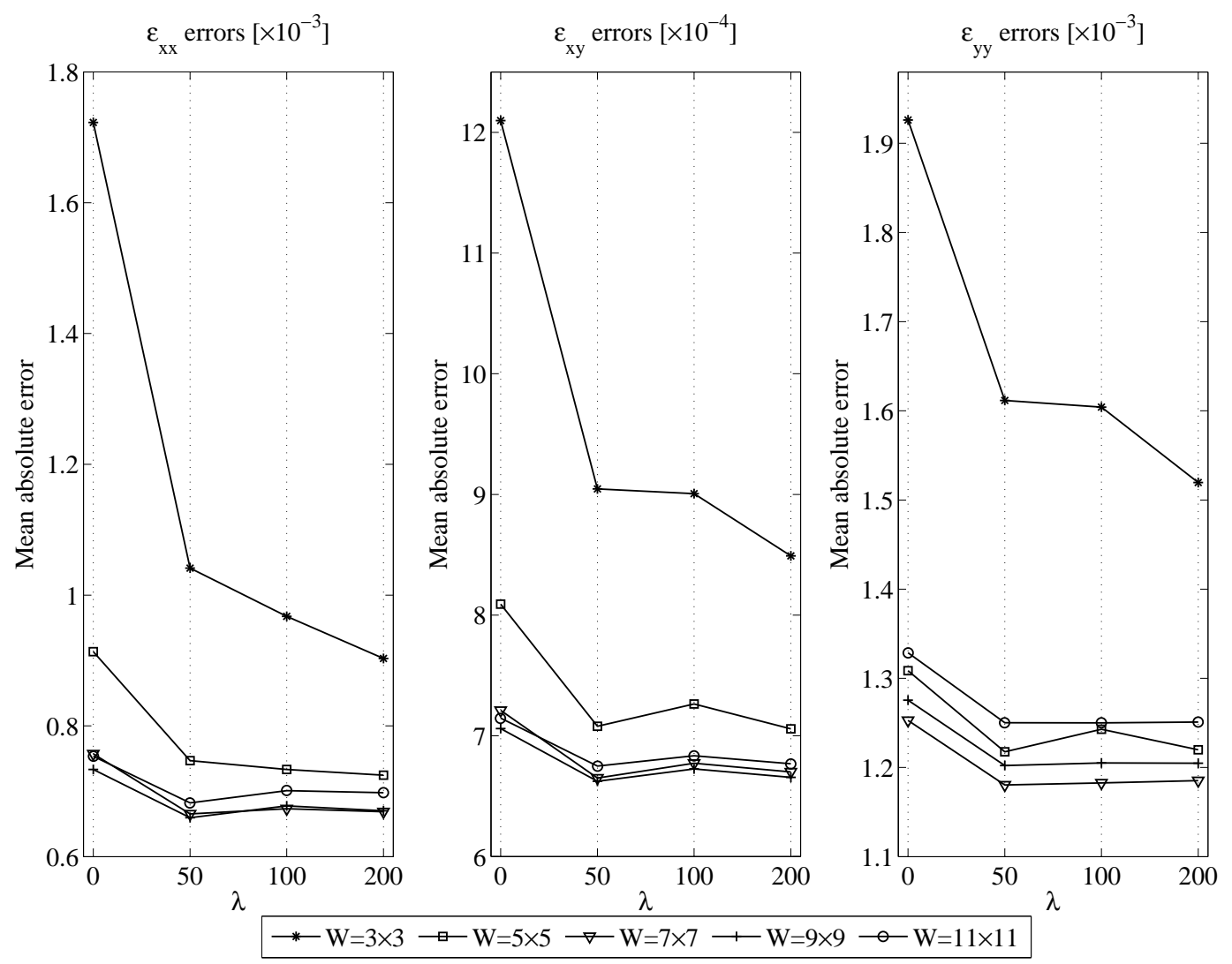

Fig. 7. Mean absolute strain errors for the horizontal (left), shear (center) and vertical (right) large (Green-Lagrange) strains as functions of the strength regularization parameter $\lambda$ and strain window size $W$ using block sizes of $15 \times 15$ pixels. F7.EPS 


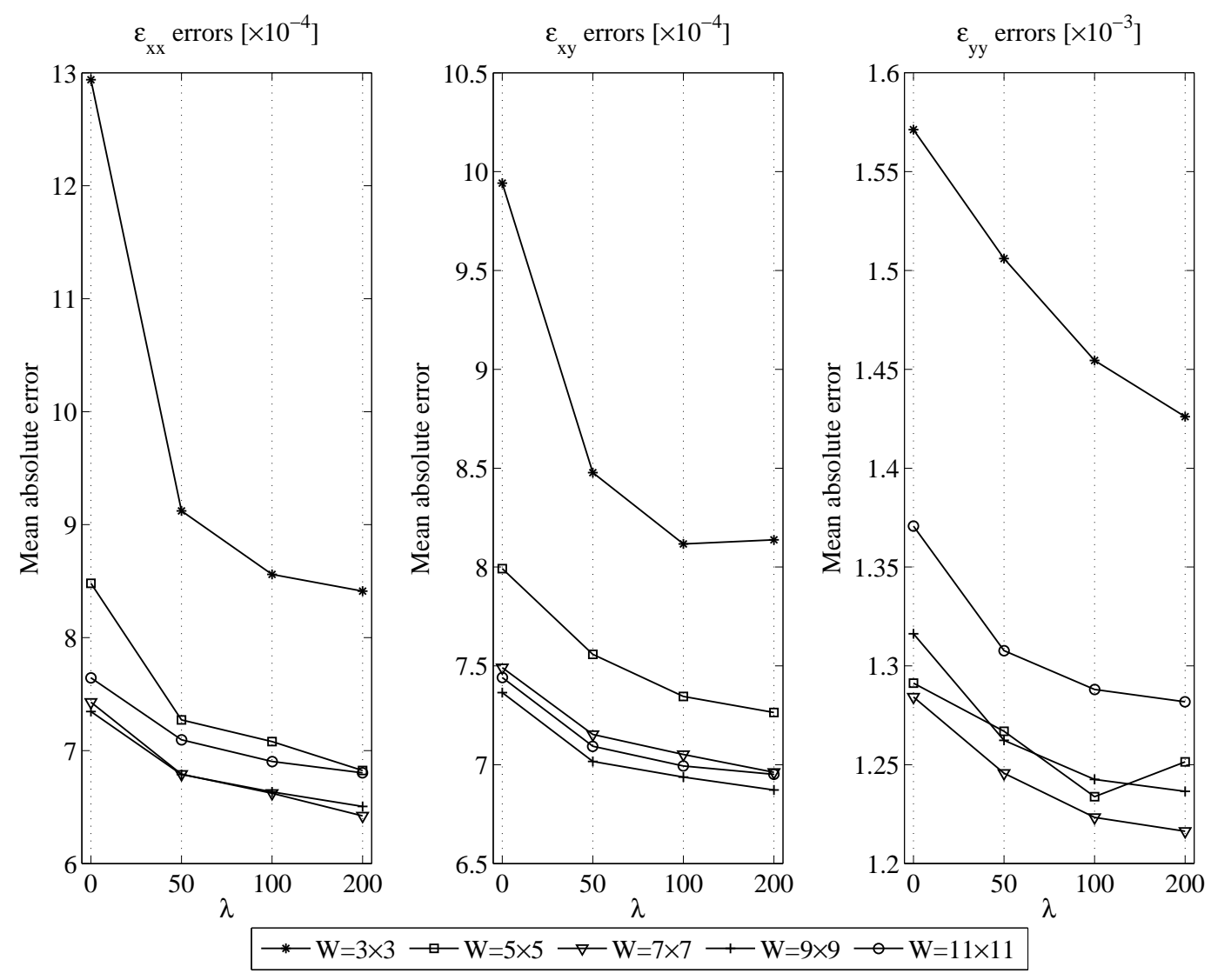

Fig. 8. Mean absolute strain errors for the horizontal (left), shear (center) and vertical (right) large (Green-Lagrange) strains as functions of the strength regularization parameter $\lambda$ and strain window size $W$ using block sizes of $21 \times 21$ pixels. F8.EPS 


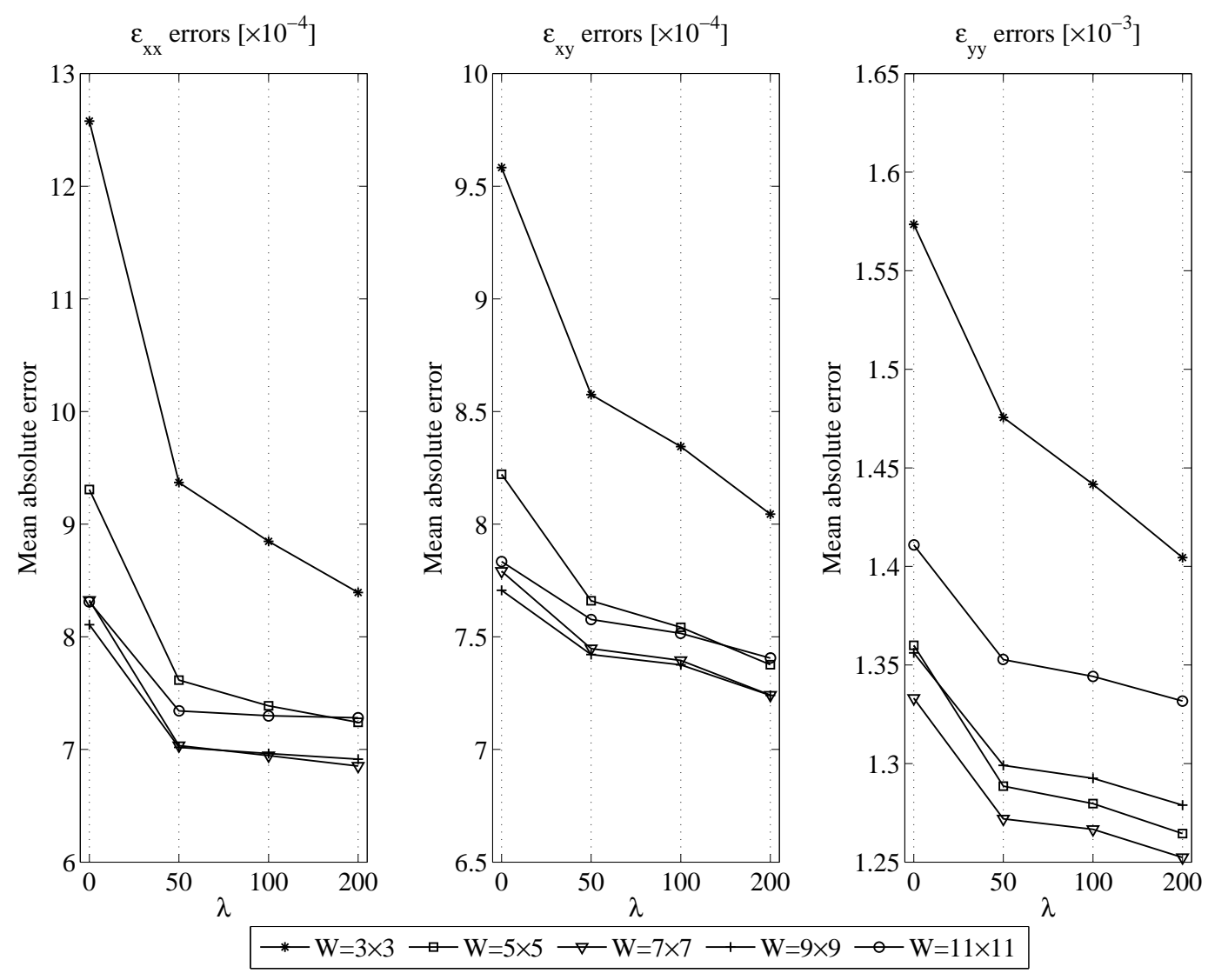

Fig. 9. Mean absolute strain errors for the horizontal (left), shear (center) and vertical (right) large (Green-Lagrange) strains as functions of the strength regularization parameter $\lambda$ and strain window size $W$ using block sizes of $27 \times 27$ pixels. F9.EPS 

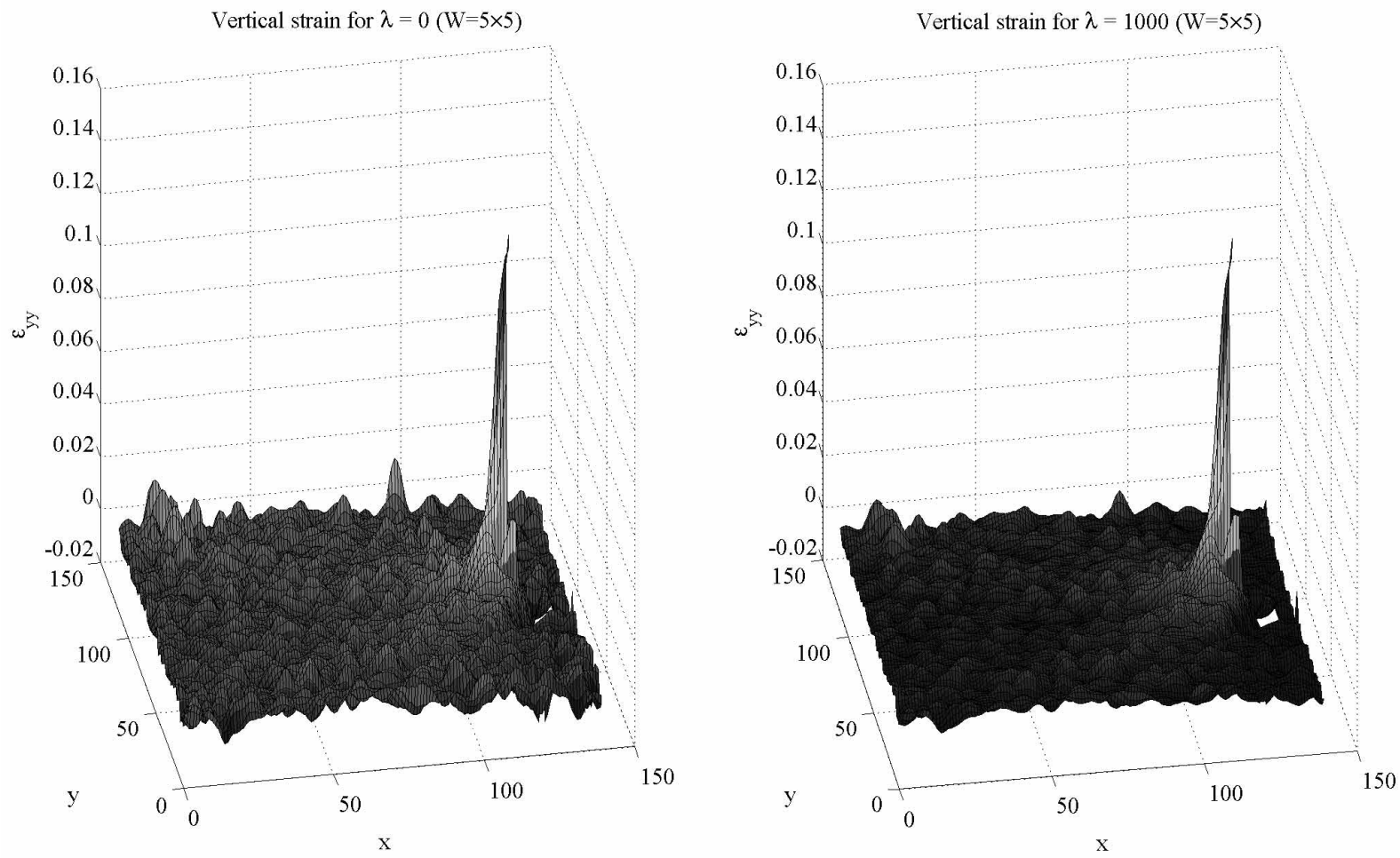

Fig. 10. Vertical strains corresponding to the third experiment. The results were obtained using $\lambda=0$ (left) and $\lambda=1000$ (right), block sizes of $27 \times 27$ pixels and a strain window of $5 \times 5$ motion vectors. F10.EPS 

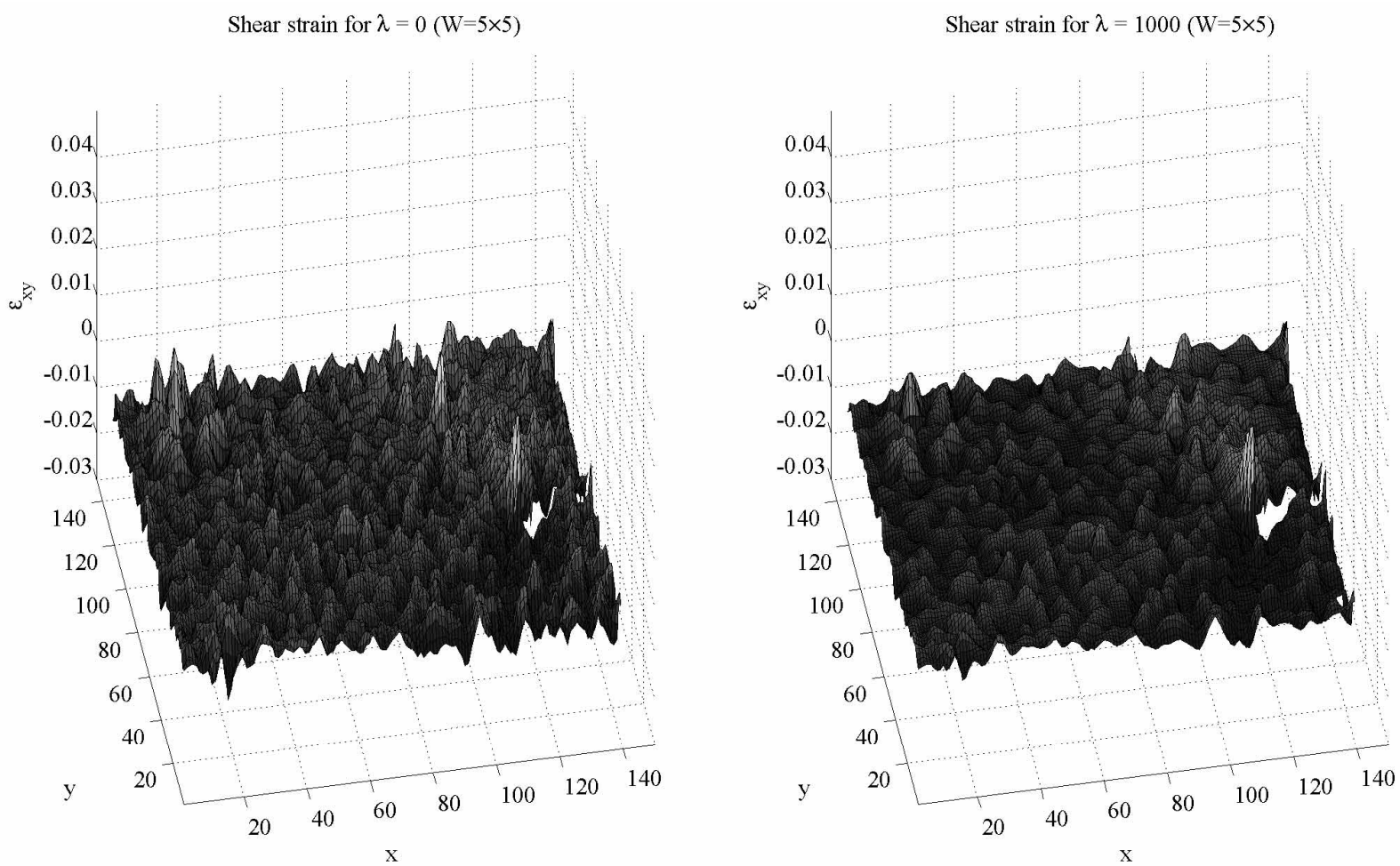

Fig. 11. Shear strains corresponding to the third experiment. The results were obtained using $\lambda=0$ (left) and $\lambda=1000$ (right), block sizes of $27 \times 27$ pixels and a strain window of $5 \times 5$ motion vectors. F11.EPS

\section{Full camera frame}
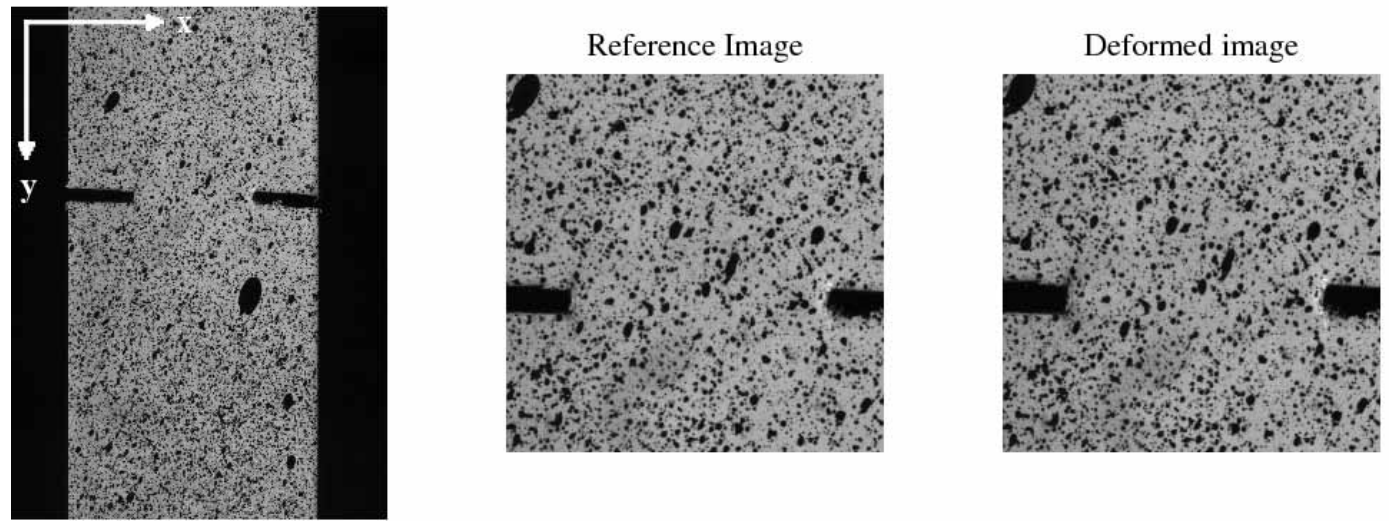

Fig. 12. The full frame captured by the camera (left), reference (center) and deformed (right) images used in the last experiment. F12.EPS 

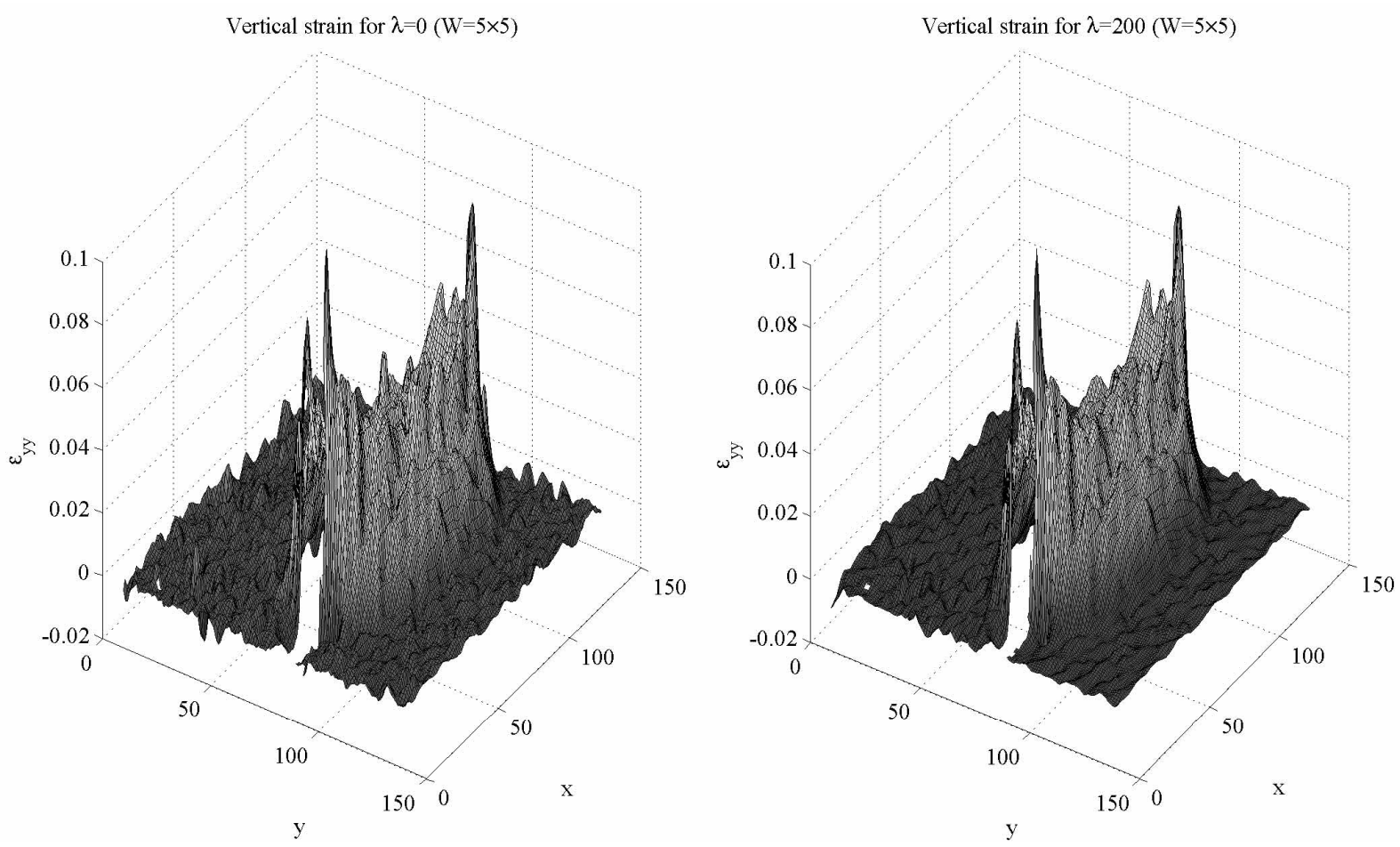

Fig. 13. Vertical strains corresponding to the fourth experiment. The results were obtained using $\lambda=0$ (left) and $\lambda=200$ (right), block sizes of $27 \times 27$ pixels and a strain window of $5 \times 5$ motion vectors. F13.EPS 

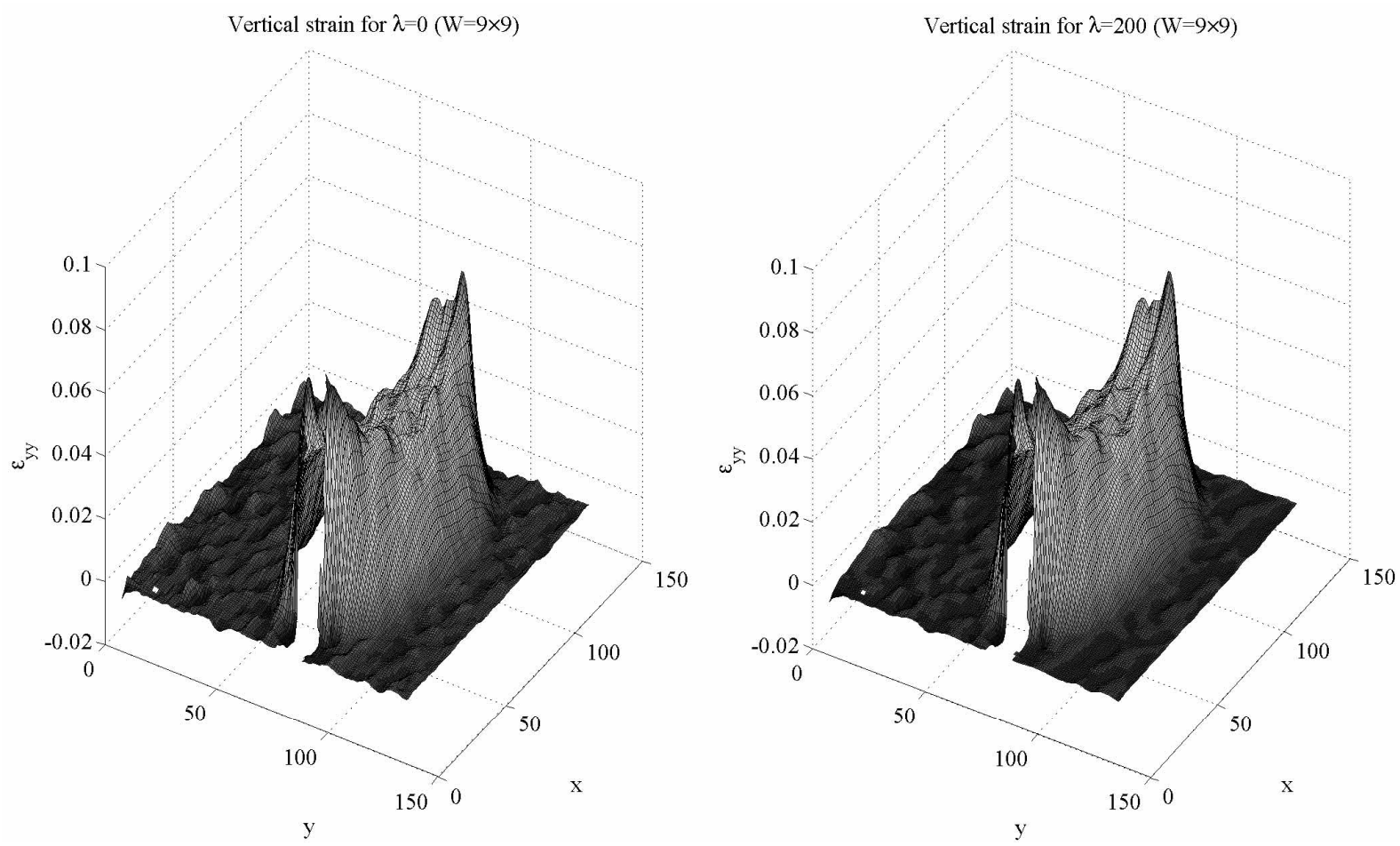

Fig. 14. Vertical strains corresponding to the fourth experiment. The results were obtained using $\lambda=0$ (left) and $\lambda=200$ (right), block sizes of $27 \times 27$ pixels and a strain window of $9 \times 9$ motion vectors. F14.EPS 

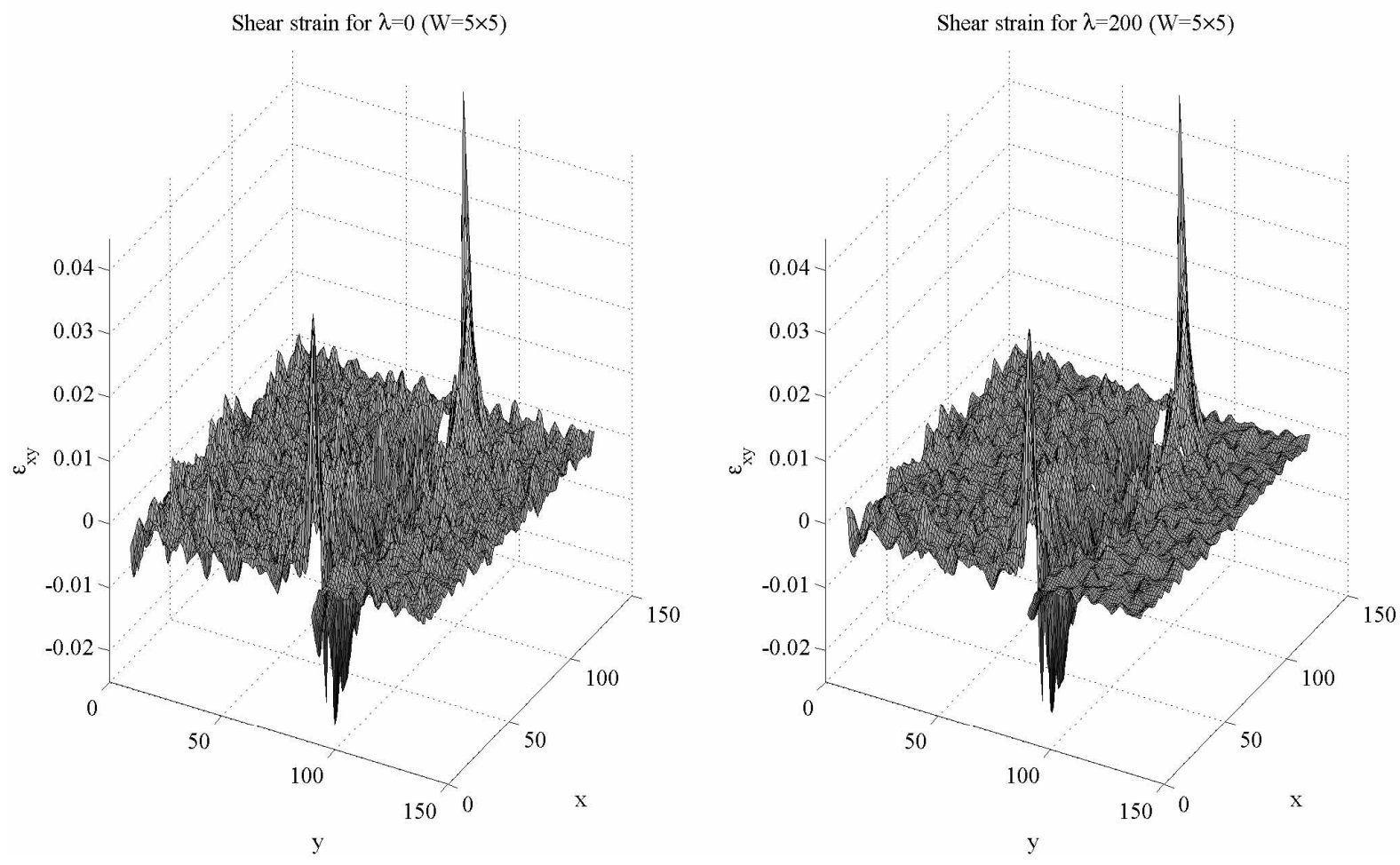

Fig. 15. Shear strains corresponding to the fourth experiment. The results were obtained using $\lambda=0$ (left) and $\lambda=200$ (right), block sizes of $27 \times 27$ pixels and a strain window of $5 \times 5$ motion vectors. F15.EPS 

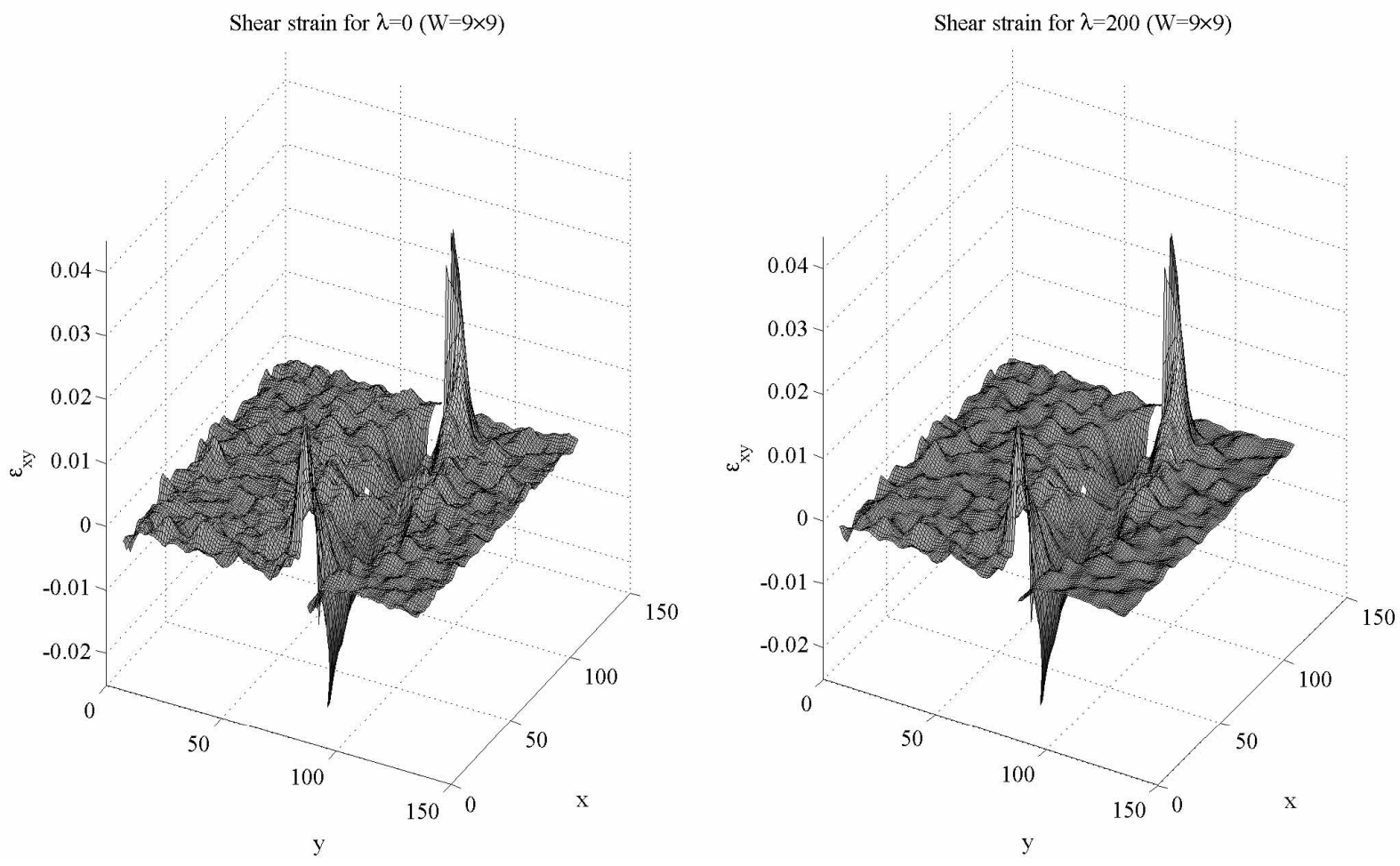

Fig. 16. Shear strains corresponding to the fourth experiment. The results were obtained using $\lambda=0$ (left) and $\lambda=200$ (right), block sizes of $27 \times 27$ pixels and a strain window of $9 \times 9$ motion vectors. F16.EPS 


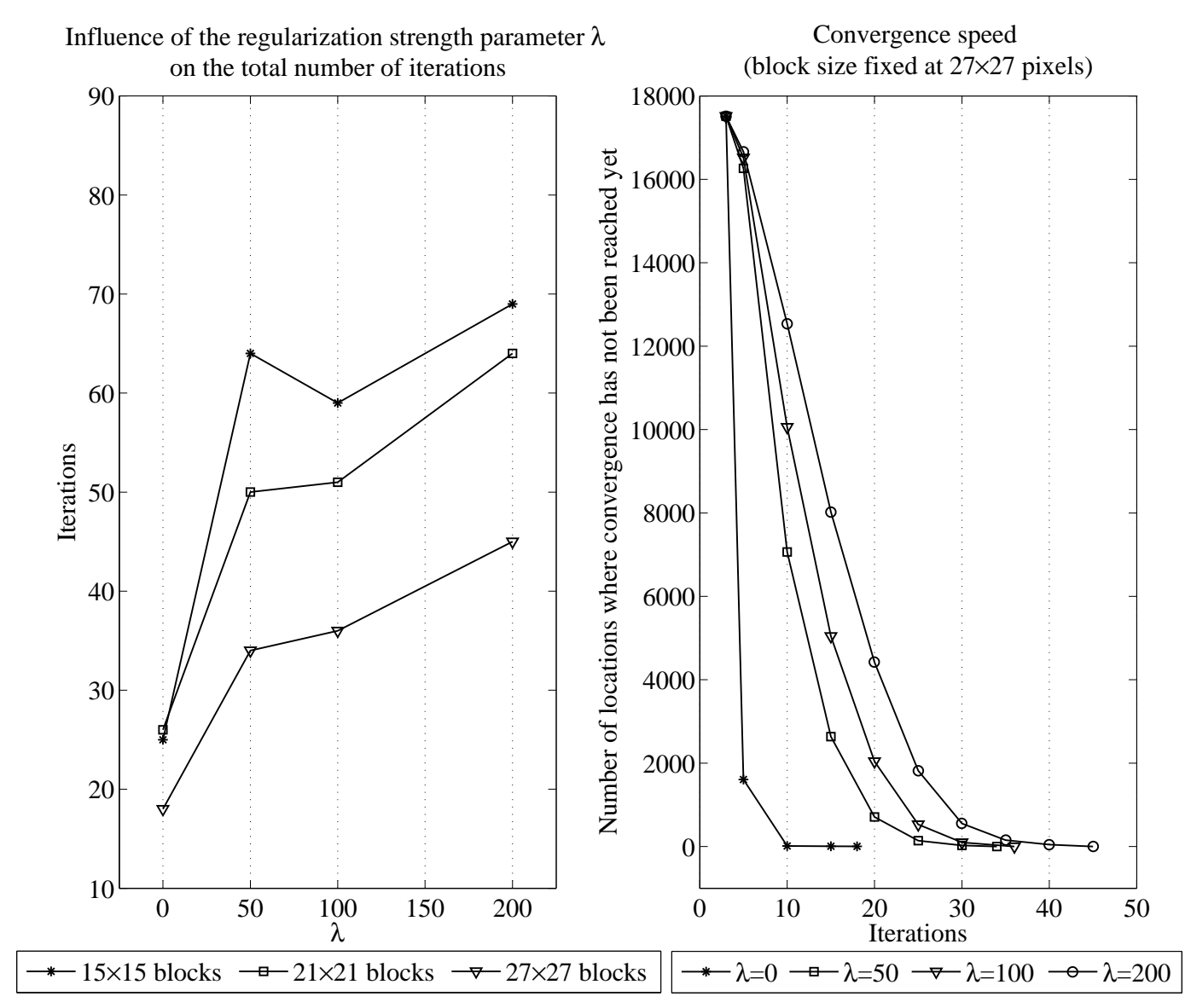

Fig. 17. Number of iterations as a function of the strength regularization parameter $\lambda$ for different block sizes (left) and the number of motion vectors that did not yet converge for a fixed block size of $27 \times 27$ pixels for different $\lambda$ values (right). F17.EPS 